\title{
In Silico Probe-Based Detection of Citrus Viruses in NGS Data
}

\author{
T. L. Jooste, M. Visser, G. Cook, J. T. Burger, and H. J. Maree
}

First, second, and fifth authors: Agricultural Research Council, Infruitec-Nietvoorbij (The Fruit, Vine and Wine Institute), Stellenbosch, South Africa; first, second, fourth, and fifth authors: Stellenbosch University, Department of Genetics, Stellenbosch, South Africa; and third author: Citrus Research International, Nelspruit, South Africa.

Accepted for publication 17 April 2017.

\begin{abstract}
The conservation of plant biosecurity relies on the rapid identification of pathogenic organisms, including viruses. With next-generation sequencing (NGS), it is possible to identify multiple viruses within a metagenomic sample. In this study, we explored the use of electronic probes (e-probes) for the simultaneous detection of 11 recognized citrus viruses. E-probes were designed and screened against raw sequencing data to minimize the bioinformatic processing time required. The e-probes were able to accurately detect their cognate viruses in simulated datasets, without any false

negatives or positives. The efficiency of the e-probe-based approach was validated with NGS datasets generated from different RNA preparations: double-stranded RNA (dsRNA) from 'Mexican' lime infected with different Citrus tristeza virus (CTV) genotypes, dsRNA from field samples, and small RNA and total RNA from grapefruit infected with the CTV T3 genotype. A set of probes was made available that is able to accurately detect CTV in sequence data regardless of the input dataset or the genotype that plants are infected with.
\end{abstract}

The past few decades have seen an increase in the introduction of foreign plant diseases along with the trading of plant material on a global scale (Gamliel and Fletcher 2008; MacDiarmid et al. 2013). There is currently no effective treatment for virus-infected plants, placing an emphasis on enhanced agricultural biosecurity and the early detection of detrimental viruses (Ochoa-Corona 2011). Presently, plant viruses are routinely detected with serological techniques such as enzyme-linked immunosorbent assay (ELISA) and nucleic acid amplification-based methods such as polymerase chain reaction (PCR) (Rodoni 2009; Sankaran et al. 2010). The application of next-generation sequencing (NGS) provides a powerful alternative to traditional diagnostic techniques and has, to date, enabled a deeper understanding of viral biodiversity (Bexfield and Kellam 2011). Although it is still too expensive to use for routine virus detection, the use of NGS for screening high-value nuclear plant material reduces the virus-detection turnaround time, increasing the effectiveness of certification schemes (MacDiarmid et al. 2013).

NGS produces large amounts of sequence data and, therefore, is subjected to computational limitations when it comes to analyzing and interpreting this data (Liu et al. 2012). In a conventional NGS virus detection approach, data will be trimmed and filtered to retain high-quality reads and then de novo assembled into contigs. These contigs are then screened against databases for homology to viruses (Adams et al. 2009). Additionally, short reads can also be mapped to reference genomes of viruses that are suspected to be present in the sample. Conventional NGS data analysis is computationally demanding, especially during the assembly and homology searching steps, and prolongs the time required for a diagnostic result.

Stobbe et al. (2013) developed a pipeline, called electronic probe (e-probe) diagnostic nucleic acid analysis (EDNA), which is

Corresponding author: H. J. Maree; E-mail address: hjmaree@sun.ac.za

T. L. Jooste and M. Visser contributed equally to the study.

*The $e$-Xtra logo stands for "electronic extra" and indicates that three supplementary tables and two supplementary protocols are published online.

(c) 2017 The American Phytopathological Society centered on utilizing probes (short sequences) unique to target pathogens for subsequent homology-based screening of raw NGS data. This targeted approach for virus detection requires less computational resources, addressing one of the major constraints accompanying NGS data analyses. In this study, we explored this alternative method for the simultaneous detection of 11 citrusinfecting viruses, using e-probes specific for each virus of interest. Additionally, we showed that an e-probe-based virus detection system for known viruses could be implemented efficiently for libraries generated from three different RNA sources: total RNA, small RNA (sRNA), and double-stranded RNA (dsRNA).

\section{MATERIALS AND METHODS}

E-probe design. E-probes were designed and optimized according to the pipeline described by Stobbe et al. (2013). Eleven citrus-infecting viruses were selected, along with near neighbor genomes (Table 1), and subjected to probe design. Near neighbors in the same genus were selected based on sequence availability and their phylogenetic relationship to the target viruses. In the instance where a target virus was the only member in a genus, a near neighbor within the same family was selected (Table 1). Mock sequence datasets (MSDs) were generated to optimize minimal probe length and the e-value threshold for similarity searching. The genome of Citrus sinensis (sweet orange) (AJPS00000000) was used as the host genome to provide background sequences. Illumina HiSeq sequencing datasets were simulated using ART software with the appropriate error model and read lengths, comparable with experimental data (Huang et al. 2012). The MSDs used for the optimization of e-probes (oMSDs) contained 10,000 simulated paired-end reads of 125 nucleotides (nts) in length. The optimization of the 11 individual probe sets was done in accordance with the workflow outlined by Stobbe et al. (2013), and entailed the screening of candidate probe sets (applying various minimum length and e-value cut-offs) against oMSDs. These oMSDs represented NGS data containing different percentages of virus sequences. All the optimization steps were performed using custom Python scripts and additionally resulted in the generation of a decoy set of probes for each of the 11 e-probe sets, by reversing the sequence of the designed target e-probes. 
Sequencing. The plant material used in this study was obtained from two greenhouse trials and field sources. The first trial was performed on the biological indicator host Mexican lime (C. aurantifolia) and the second on commercial Marsh grapefruit (C. paradisi). Virus-free buds of Marsh plants were grafted onto rough lemon rootstocks (C. jambhiri) and Mexican lime plants were grown from seed. After 6 months, Citrus tristeza virus (CTV) single-variant sources of the different genotypes were used to inoculate the Mexican lime plants in the first trial (Table 2). Five plants (biological replicates) were inoculated for each CTV genotype. Replicate plants from trial 1 were pooled to provide sufficient material for nucleic acid extraction 1 year postinoculation. The grapefruit plants in the second trial were inoculated with CTV genotype T3 (Table 2). The success of CTV inoculation was confirmed by reverse-transcription (RT)-PCR (Cook et al. 2016; Supplementary Table S1). Six additional field samples were obtained from the Hoedspruit region, Limpopo Province, South Africa.

dsRNA was extracted from the pooled material of Mexican lime plants (trial 1) using an affinity chromatography method (Burger and Maree 2015). This method consisted of two cycles of cellulose affinity chromatography using MN 2100 (Macherey-Nagel) cellulose powder. Plant phloem material was used at $12 \mathrm{~g} /$ extraction. The dsRNA suspension was subjected to RNase and DNase treatment using RQ1 RNase-free DNase (Promega Corp.) and T1 RNase (Roche) according to the manufacturers' protocols. The quality and

TABLE 1. Electronic probe design for 11 citrus infecting viruses

\begin{tabular}{|c|c|c|c|c|c|c|}
\hline \multicolumn{4}{|c|}{ Target pathogen } & \multicolumn{3}{|c|}{ Near neighbor } \\
\hline Genus & Virus & Abbrev & Accession & Genus & Virus & Accession \\
\hline Closterovirus & Citrus tristeza virus & CTV & EU937519.1 & Closterovirus & Grapevine leafroll associated virus 2 & NC_007448 \\
\hline Ophiovirus & Citrus psorosis virus & CPsV & $\begin{array}{l}\text { NC_006314 } \\
\text { NC_006315 } \\
\text { NC_006316 }\end{array}$ & Ophiovirus & Mirafiori lettuce big-vein & $\begin{array}{l}\text { NC_004779 } \\
\text { NC_004781 } \\
\text { NC_004782 }\end{array}$ \\
\hline Capilovirus & Citrus tatter leaf virus ${ }^{a}$ & CTLV & JX $\overline{4} 16228$ & Capilovirus & Cherry virus $A$ & NC_003689 \\
\hline Ilarvirus & Citrus variegation virus & CVV & $\begin{array}{l}\mathrm{NC} \_009536 \\
\mathrm{NC} \_009537 \\
\mathrm{NC} \_009538\end{array}$ & Ilarvirus & Citrus leaf rugose virus & $\begin{array}{l}\mathrm{NC} \_003546 \\
\mathrm{NC} \_003547 \\
\mathrm{NC} \_003548\end{array}$ \\
\hline Badnavirus & Citrus yellow mosaic virus & CYMV & NC_003382 & Badnavirus & Cacao swollen shoot virus & NC_001574 \\
\hline Ilarvirus & Citrus leaf rugose virus & CiLRV & $\begin{array}{l}\text { NC_003546 } \\
\text { NC_003547 } \\
\text { NC_003548 }\end{array}$ & Ilarvirus & Citrus variegation virus & $\begin{array}{l}\text { NC_009536 } \\
\text { NC_009537 } \\
\text { NC_009538 }\end{array}$ \\
\hline Citrivirus & Citrus leaf blotch virus & CLBV & NC_003877 & Prunevirus & $\begin{array}{l}\text { Apricot vein clearing associated virus, } \\
\text { isolate VC }\end{array}$ & NC_023295 \\
\hline Cilevirus & Citrus leprosis virus $C$ & CiLV-C & NC_008169 & Higrevirus & $\begin{array}{l}\text { Hibiscus green spot virus } 2 \\
\text { isolate WAI } 1-1\end{array}$ & NC_016141 \\
\hline Mandarivirus & Indian citrus ringspot virus & ICRSV & $\begin{array}{l}\text { NC_008170 } \\
\text { NC_003093 }\end{array}$ & Mandarivirus & $\begin{array}{l}\text { Citrus yellow vein clearing } \\
\text { virus - isolate } \mathrm{CQ}\end{array}$ & $\begin{array}{l}\text { NC_016143 } \\
\text { NC_026592 }\end{array}$ \\
\hline Mandarivirus & $\begin{array}{l}\text { Citrus yellow vein clearing virus, } \\
\text { isolate CQ }\end{array}$ & CYVCV & NC_026592 & Mandarivirus & Indian citrus ringspot virus & NC_003093 \\
\hline Sadwavirus & Satsuma dwarf virus & SDV & $\begin{array}{l}\text { NC_003785 } \\
\text { NC_003786 }\end{array}$ & Unassigned & Strawberry mottle virus & $\begin{array}{l}\text { NC_003445 } \\
\text { NC_003446 }\end{array}$ \\
\hline
\end{tabular}

a Isolate of Apple stem grooving virus.

TABLE 2. Virus-infected plant material used in study

\begin{tabular}{|c|c|c|c|}
\hline Sample ID & Source ${ }^{a}$ & Cultivar ${ }^{\mathrm{b}}$ & Library type $^{c}$ \\
\hline SS_RB1 & B389-1 (RB1) & MXL & dsRNA (RNASeq) \\
\hline SS_RB2 & B389-4 (RB2) & MXL & dsRNA (RNASeq) \\
\hline SS_LMS & LMS 6-6 (HA16.5) & MXL & dsRNA (RNASeq) \\
\hline SS_T68 & GFMS 12-8 (T68) & MXL & dsRNA (RNASeq) \\
\hline SS_VT & Maxi x3 (VT) & MXL & dsRNA (RNASeq) \\
\hline T1_RB1 & B390-5 (RB1) & MXL & dsRNA (RNASeq) \\
\hline T1_RB2 & B389-4 (RB2) & MXL & dsRNA (RNASeq) \\
\hline T1_LMS & LMS 6-6 (HA16.5) & MXL & dsRNA (RNASeq) \\
\hline T1_T68 & GFMS 12-8 (T68) & MXL & dsRNA (RNASeq) \\
\hline T1_VT & Maxi x 3 (VT) & MXL & dsRNA (RNASeq) \\
\hline T2_HVK4 & $\mathrm{T} 3$ & GF-M & sRNA \\
\hline T2_HVK5 & T3 & GF-M & sRNA \\
\hline T2_HVK6 & T3 & GF-M & sRNA \\
\hline T2_HVK16 & $\mathrm{T} 3$ & GF-M & Total RNA \\
\hline T2_HVK17 & $\mathrm{T} 3$ & GF-M & Total RNA \\
\hline T2_HVK18 & $\mathrm{T} 3$ & GF-M & Total RNA \\
\hline UNK_01 & Field samples, status unknown & Val-D & dsRNA (RNASeq) \\
\hline UNK_02 & Field samples, status unknown & Val-D & dsRNA (RNASeq) \\
\hline UNK_03 & Field samples, status unknown & Val-D & dsRNA (RNASeq) \\
\hline UNK_04 & Field samples, status unknown & SO-MV & dsRNA (RNASeq) \\
\hline UNK_05 & Field samples, status unknown & SO-MV & dsRNA (RNASeq) \\
\hline UNK_06 & Field samples, status unknown & SO-MV & dsRNA (RNASeq) \\
\hline
\end{tabular}

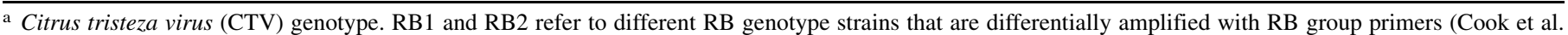
2016).

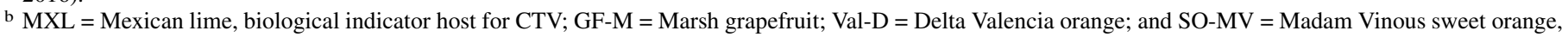
indicator host for Citrus psorosis virus.

c Double-stranded RNA (dsRNA), small RNA (sRNA), and total RNA. 
quantity of the dsRNA was evaluated through visualization on a $1 \%$ (wt/vol) Tris-acetate-EDTA agarose gel stained with ethidium bromide. Total RNA was extracted from three CTV-infected grapefruit plants (trial 2) using an adapted version of the low molecular weight RNA extraction method described by Carra et al. (2007). The method involves extracting total RNA from $2 \mathrm{~g}$ of bark shavings using a cetyltrimethylammonium bromide (CTAB) extraction buffer $(2 \%$ [wt/vol] CTAB, 2.5\% [wt/vol] PVP-40, $100 \mathrm{mM}$ Tris-HCL [pH8], $2 \mathrm{M} \mathrm{NaCl}, 25 \mathrm{mM}$ EDTA [pH8], and 3\% [vol/vol] $\beta$-mercaptoethanol) and isopropanol precipitation. The polyethylene glycol precipitation step was omitted and additional chloroform extractions were performed to remove polysaccharides.

Library preparations for dsRNA (trial 1 and field samples) were performed using an adapted Illumina TruSeq RNA sample preparation protocol (Burger and Maree 2015). These libraries were sequenced in a paired-end $(2 \times 125 \mathrm{nt})$ run on an Illumina HiSeq2500 platform at the Agricultural Research Council's Biotechnology Platform in Pretoria, South Africa. Total RNA samples (trial 2) were used to prepare two separate types of sequencing libraries: an Illumina Small RNA TruSeq protocol was followed to allow for sRNA sequencing and a TruSeq Stranded messenger RNA (mRNA) protocol for whole-transcriptome sequencing. Both libraries were sequenced on an Illumina HiSeq2500 at Fasteris, with single reads $(1 \times 50 \mathrm{nt})$ for sRNA libraries and paired-end reads $(2 \times$ $125 \mathrm{nt}$ ) for ribosomal RNA-depleted transcriptome libraries.

E-probe-based virus detection. The virus status of a sample is based on the presence of reads in a dataset that are similar to the e-probes for a specific virus. Virus-specific e-probes and corresponding decoys were screened against sequence data, and the BLASTn results were used to score each probe in both the target and decoy sets (Stobbe et al. 2013). The scores of the e-probe sets were statistically compared with those of the decoy sets. The normality (Shapiro-Wilk test) and population variance of the two datasets were first determined. If the two datasets were normally distributed, either a student $t$ test (same variance) or a Welch's $t$ test (difference in variance) was performed. A nonparametric test (Wilcoxon Ranksum) was performed in the instance where the datasets were not normally distributed. A $P$ value $\leq 0.05$ indicated a positive call whereas a $P$ value $>0.1$ would indicate the absence of the specific virus (Stobbe et al. 2013). A third outcome was presented for samples with a $P$ value $\leq 0.1$ and $>0.05$, because they were only "suspected" to be positive (Stobbe et al. 2013).

Detection using simulated datasets. In silico testing of the e-probe sets designed for the 11 viruses in this study was performed with evaluation-MSDs (eMSDs) that were generated using ART Illumina simulation software (Huang et al. 2012) in the same way as previously described and contained both host and virus genome segments. The datasets used for probe evaluation consisted of 1 million paired-end reads, each $125 \mathrm{nts}$ in length, with a virus abundance level of $1 \%$. Single-infection datasets were constructed for each of the 11 viruses using the reference genome. Each eMSD served as a reference dataset against which virus-specific e-probe sets (including decoys) were screened using BLASTn. Additionally, probe sets were evaluated for their ability to detect different isolates of each target virus species. GenBank accessions of full genomes of the different virus isolates were used to construct eMSDs in the same way as previously described.

Detection using NGS datasets. The raw data obtained from NGS of plant samples were screened with the designed target e-probe and corresponding decoy sets for all 11 viruses using BLASTn. In the case of paired-end data, the two read files were pooled to create one input dataset to be used for virus detection. The infection status of the datasets were determined using the probe based detection system. De novo assemblies and similarity searches were used, along with RT-PCR (Cook et al. 2016), to confirm these results. Individual probes within the e-probe set designed for CTV detection were further evaluated for their performance with sRNA and transcriptome library types. The sequence data of three representatives of each sequence library type (sRNA and transcriptome) was subjected to the random sampling of 1 million reads 100 times for each sample. The e-probes were screened against the 100 subsampled datasets and, in each instance, the number of times a specific probe hit was noted. The number of hits for a specific probe was averaged across the three samples from a specific dataset and plotted. The two sequencing libraries differed in the RNA species that were sequenced, read length, and the number of viral sequences present in each data set. To evaluate the influence of some of these differences, additional subsampling experiments were conducted to assess the performance of the CTV probes. The influence of read length was evaluated by only using the first $23 \mathrm{nts}$ of the forward reads of the transcriptome datasets. The impact of the percentage of virus-specific reads was evaluated by increasing the amount sRNA data subsampled to 5.7 million reads, to be comparable with the transcriptome data.

\section{RESULTS}

E-probe design and evaluation. Virus-specific e-probes of varying minimum lengths were generated for the 11 viruses in this study by comparing each virus to a near neighbor (Table 1 ). The number of candidate probes generated ranged from 62 to 211 per virus species. No correlation was seen between the size of the genome and the number of candidate probes generated for all of the target viruses.

During probe length optimization, the candidate probe sets of different minimum lengths showed $100 \%$ precision for all the viruses targeted. This was also reported during the evaluation of the EDNA pipeline (Stobbe et al. 2013). The number of matches was indirectly proportional to the minimum length of the e-probes. The effect of varying the minimum e-probe lengths showed that the probe sets with longer minimum lengths (those with 120 and $140 \mathrm{nt}$ ) generated the lowest number of hits (if any) in the very low pathogen percentage oMSDs. The e-probe sets of each virus with a minimum length of 20 nts were selected for e-value threshold determination, because they had the highest number of matches and displayed precision of $100 \%$ when screened against oMSDs of varying virus concentrations (including those representing very low virus abundance levels).

The use of different e-values was implemented to investigate the occurrence of potential false positives. However, no differences were seen because all of the viruses were detected in their respective oMSDs, at each e-value $\left(10^{-3}\right.$ to $\left.10^{-9}\right)$, even in the very low pathogen abundance datasets. Because no false positives were detected at an e-value cut-off of $1 \times 10^{-3}$, this value was selected for candidate specificity screening as well as for screening e-probe sets against NGS data. National Center for Biotechnology Information (NCBI) filtering of the optimized candidate probes decreased the number of probes per virus, resulting in the number of final e-probes ranging from 51 to 209 per virus.

The single-infection eMSDs all tested positive only for the presence of the specific virus species used in its construction, with each probe set being able to identify the target virus and all its corresponding isolates (Supplementary Table S2). Indian citrus ringspot virus probe hits were observed in the eMSDs representing infection with Citrus yellow vein clearing virus isolates, and vice versa. This reciprocal interaction was also seen between Citrus leaf rugose virus and Citrus variegation virus. These hits, however, did not generate a signal significant enough to shift the $P$ value $<0.01$, or even 0.05 , and, therefore, the final diagnostic result remained negative.

E-probe-based virus detection in NGS data. The raw sequence files obtained from the CTV-infected trial plants and field samples were used as reference datasets to screen all of the probe sets. Validation of the probe-based detection system revealed that all the samples, including the field samples, were positive for CTV (Supplementary Table S3). The set of 209 CTV probes designed 
from a single genotype was able to detect the presence of all the other CTV genotypes used in the study. The number of probes that hit differed from sample to sample; however, more importantly, no decoys hit in any of the instances. The remaining 10 probe sets were used to screen all of the samples and detected CPsV in only one field sample. The results were confirmed with de novo assembly-based similarity searches and RT-PCR.

To evaluate individual probe performance, three datasets, representing each of the two different sequencing libraries, were selected and the CTV probe set was screened against them
A

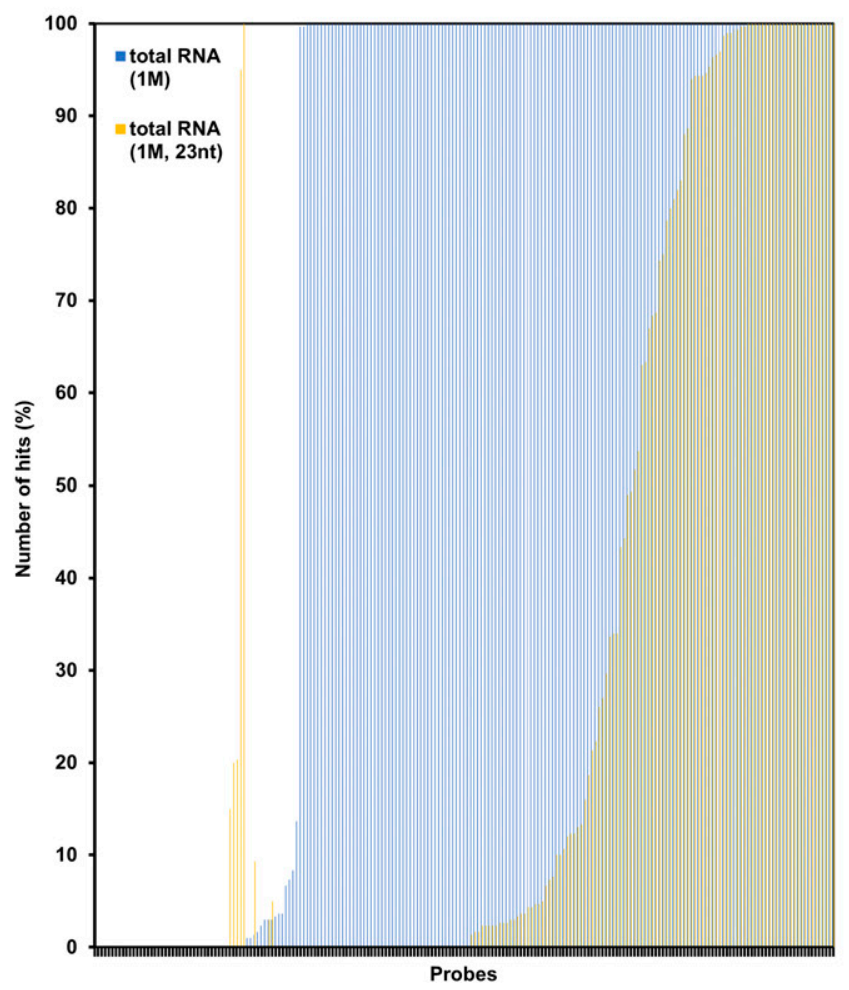

B

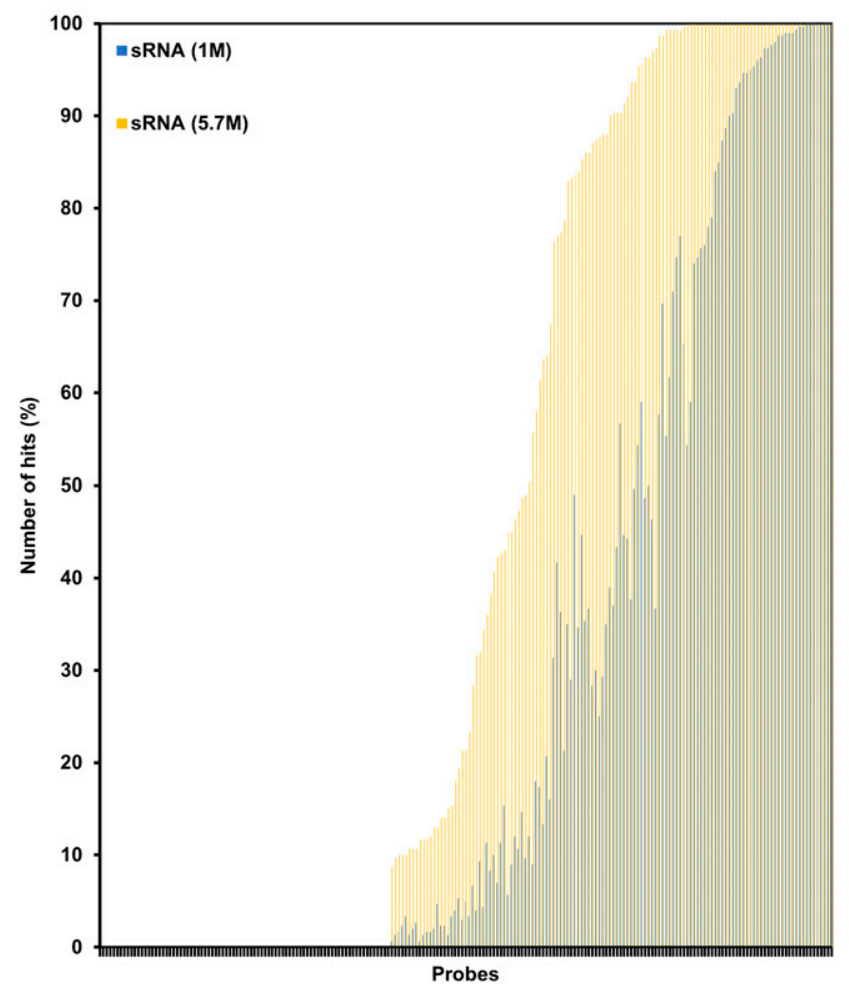

Fig. 1. Performance evaluation of Citrus tristeza virus electronic probes across different library types. A, Illustration of probe performance for transcriptome data (subsample size of 1 million reads) with full-length paired reads of 125 nucleotides (nts) compared with the first 23 nts of the forward reads of the same samples. Results are first arranged according to number of hits against the full-length transcripts (lowest to highest), followed by arrangement according to the number of hits against the trimmed reads (lowest to highest). B, Probe performance against small RNA (sRNA) data with a subsample size of 1 million compared with a subsample size of 5.7 million. Results are first arranged according to number of hits against the 1 million read subsamples (lowest to highest), followed by arrangement according to the number of hits against 5.7 million read subsamples (lowest to highest).

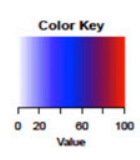

A

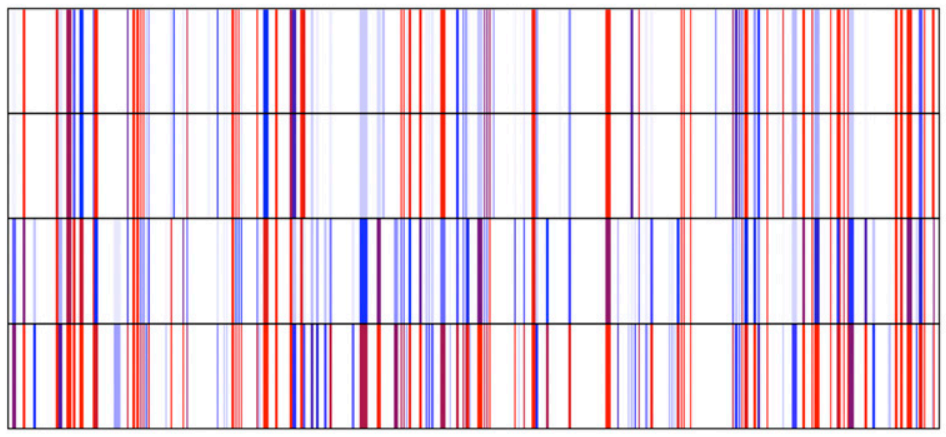

total RNA (1M, 23nt)

total RNA (1M)

$\operatorname{sRNA}(1 \mathrm{M})$

SRNA (5.7M)

B

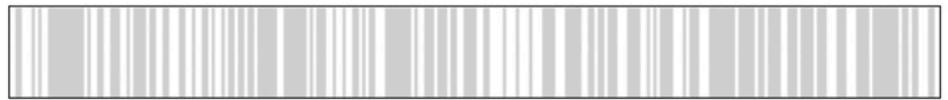

Probes

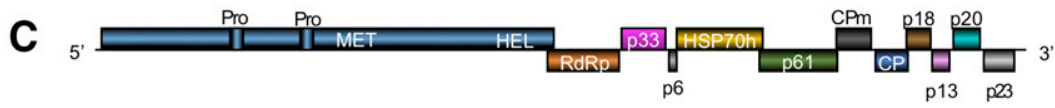

CTV

Fig. 2. Evaluation of Citrus tristeza virus (CTV) probe performance across different library types. A, Heat map of the number of times a specific probe hit in the four datasets; sRNA = small RNA, respectively. B, CTV genomic regions covered by the 209 electronic probes and C, CTV genome organization with 11 open reading frames. 
(Fig. 1). Within the transcriptome dataset, approximately $71 \%$ of the total probes were detected in all 100 subsets (i.e., $100 \%$ of the time) whereas the remaining probes hit $<20 \%$ of the time or not at all (Fig. 1A). However, this trend was not observed within the sRNA datasets, with a mere $3.8 \%$ of the probes hitting $100 \%$ of the time and $39.7 \%$ of the probes not hitting at all. Increasing the subsample size of the sRNA datasets yielded a similar profile, with $20 \%$ of the probes now being detected in all 100 subsets and $36 \%$ of the probes not generating any hits at all (Fig. 1B). The shortening of transcriptome data reads led to a significant drop in the number of probes hitting $100 \%$ of the time (12\% of the total probes), displaying a probe performance profile similar to that seen with sRNA data (Fig. 1A). Taking into consideration the genomic positions of the probes, mapping them onto the CTV genome revealed that they extend over the complete genome and are not localized to a specific area (Fig. 2). Probes that were not detected across both library types were not removed from the final probe set, because they might be homologous to a region on the virus genome that is not represented in this specific sequence data.

\section{DISCUSSION}

The e-probe bioinformatic approach proposed by Stobbe et al. $(2013,2014)$ was evaluated in this study for its ability to detect multiple citrus viruses simultaneously in NGS data. Previously, the validation of this approach used a series of simulated datasets with representatives of multiple taxonomic groups of pathogens in a general plant background ( $V$. vinifera) (Stobbe et al. 2013). In the present study, e-probes were designed for the simultaneous detection of 11 citrus-infecting viruses of global economic importance in simulated NGS datasets. The validation was further extended to RNASeq datasets of CTV-infected plants and included individual probe performance evaluation across two different sequencing library types (sRNA and transcriptome).

E-probes were successfully developed for the 11 viruses by comparing each virus to its nearest neighbor (Table 1). Evaluation of these e-probe sets in searches against simulated datasets containing all the accessible virus isolates showed that this probebased detection approach was effective in detecting different isolates of the same citrus virus. However, this observation may not be true for more genetically divergent virus species, and caution should be taken when selecting a reference genome for e-probe design (Visser et al. 2016). Instances also occurred where e-probes matched to a nontarget virus. Interestingly, in these cases, the nontarget virus was also the virus used as near neighbor during probe design. Despite the fact that the BLASTn filtering step against the NCBI's nucleotide database was unable to remove individual nonspecific probes, all probe sets generated no false positive or even suspected results, indicating the specificity and sensitivity of these probes when used as sets.

The e-probe-based detection system was validated for CTV by employing the probe set constructed from the VT genotype (EU937519) genome sequence in searches of NGS data obtained from plant samples. This e-probe set positively detected CTV in sequence data from dsRNA of plants infected with CTV genotypes other than VT, again demonstrating that this probe set is able to detect CTV infection regardless of the genotype. There was no instance where all 209 probes hit in the plant sequence data, including the sample infected with a VT genotype. This can be due to isolate variation or the length of the NGS reads. These datasets were also screened with the remaining 10 probe sets, which revealed the presence of Citrus psorosis virus in one of the field samples in addition to CTV.

Individual CTV probes were further evaluated in terms of their performance across two different NGS data types. In both instances, the complete probe set was able to accurately detect the presence of the virus. However, the ability of each individual probe to hit a virus- derived sequence $100 \%$ of time in the sRNA datasets was markedly lower than in transcriptome data (Fig. 1). No significant correlation between probe performance and its coordinates on the CTV genome was observed (Fig. 2).

The variation in the performance of the two library types can be attributed to fundamental differences in the sequence data. The percentage of virus-derived sRNA sequences is dependent on the host defense response, unlike the virus-associated reads in the transcriptome data. The datasets used all contained the same number of sequence reads but the percentages of virus-derived reads were 0.69 and $2.4 \%$ for sRNA and transcriptome data, respectively. Increasing the sRNA subsample size to make the virus-derived data comparable with that of the transcriptome data showed a similar decrease in probe performance (Fig. 1B). This suggests that the percentage of virus-derived data in NGS datasets has a limited effect on the probe performance.

The influence of read length also becomes evident because the reads generated by sRNA sequencing are much smaller (approximately $22 \mathrm{nts}$ ) than those in transcriptome sequencing (125 nts), which may influence the BLAST-based similarity search. Evaluating the influence of shortened transcriptome reads on probe performance showed a performance profile similar to that seen with the sRNA datasets (Fig. 1A). This highlighted the significant impact of read length on the performance of the probes, with longer reads being more favorable.

This e-probe-based bioinformatic pipeline shifts the focus of traditional metagenomic data analysis from attempting to establish a complete profile of all organisms within a given sample to screening for multiple known viruses simultaneously. Searching for the presence of sequences specific to target viruses not only alleviates the computational workload but also reduces the time required to make a diagnostic call. This is particularly useful in agricultural sectors where products or plant materials require specific viral tests prior to imports. Our results show that an e-probe-based assay for the detection of known viruses in a complex host such as citrus is effective not only with the use of simulated data but also with different types of NGS data. The statistical tests employed add confidence to the diagnostic pronouncement, unlike the often subjective, user-based review process involved in conventional NGS approaches. Furthermore, the flexibility of this approach allows for it to be automated and adjusted for viruses infecting multiple alternate hosts (Visser et al. 2016). The probes generated, along with all the appropriate Python scripts used in this study, are freely available with the user-friendly software Truffle (https:// sourceforge.net/projects/truffle/).

\section{ACKNOWLEDGMENTS}

This study was funded by Citrus Research International, the Technology and Human Resources for Industry Programme (Department of Trade and Industry, South Africa, Grant TP13081327563), and the National Research Foundation (NRF). Opinions expressed and conclusions drawn are those of the authors and not necessarily to be attributed to the NRF.

\section{LITERATURE CITED}

Adams, I. P., Glover, R. H., Monger, W. A., Mumford, R., Jackeviciene, E., Navalinskiene, M., Samuitiene, M., and Boonham, N. 2009. Next-generation sequencing and metagenomic analysis: A universal diagnostic tool in plant virology. Mol. Plant Pathol. 10:537-545.

Bexfield, N., and Kellam, P. 2011. Metagenomics and the molecular identification of novel viruses. Vet. J. 190:191-198.

Burger, J. T., and Maree, H. J. 2015. Metagenomic next-generation sequencing of viruses infecting grapevines. Methods Mol. Biol. 1302:315-330.

Carra, A., Gambino, G., and Schubert, A. 2007. A cetyltrimethylammonium bromide-based method to extract low-molecular-weight RNA from polysaccharide-rich plant tissues. Anal. Biochem. 360:318-320.

Cook, G., van Vuuren, S. P., Breytenbach, J. H. J., Burger, J. T., and Maree, H. J. 2016. Expanded strain-specific RT-PCR assay for differential 
detection of currently known Citrus tristeza virus strains: A useful screening tool. J. Phytopathol. 164:847-851.

Gamliel, A., and Fletcher, J. 2008. Crop biosecurity: Containment and eradication of invasive pathogens. Pages 71-90 in: Crop Biosecurity: Assuring our Global Food Supply. Springer, The Netherlands.

Huang, W., Li, L., Myers, J. R., and Marth, G. T. 2012. ART: A nextgeneration sequencing read simulator. Bioinformatics 28:593-594.

Liu, L., Li, Y., Li, S., Hu, N., He, Y., Pong, R., Lin, D., Lu, L., and Law, M. 2012. Comparison of next-generation sequencing systems. J. Biomed. Biotechnol. 2012: Article 251364.

MacDiarmid, R., Rodoni, B., Melcher, U., Ochoa-Corona, M., and Roossinck, M. 2013. Biosecurity implications of new technology and discovery in plant research. PLoS Pathog. 9:e1003337.

Ochoa-Corona, F. M. 2011. Biosecurity, microbial forensics and plant pathology: Education challenges, overlapping disciplines and research needs. Australas. Plant Pathol. 40:335-338.
Rodoni, B. 2009. The role of plant biosecurity in preventing and controlling emerging plant virus disease epidemics. Virus Res. 141:150-157.

Sankaran, S., Mishra, A., Ehsani, R., and Davis, C. 2010. A review of advanced techniques for detecting plant disease. Comput. Electron. Agric. 72: $1-13$.

Stobbe, A. H., Daniels, J., Espindola, A. S., Verma, R., Melcher, U., Ochoa-Corona, F., Garzon, C., Fletcher, J., and Schneider, W. 2013. E-probe diagnostic nucleic acid analysis (EDNA): A theoretical approach for handling next generation sequence data for diagnostics. J. Microbiol. Methods 94:356-366.

Stobbe, A. H., Schneider, W. L., Hoyt, P. R., and Melcher, U. 2014. Screening metagenomic data for viruses using the e-probe diagnostic nucleic acid assay. Phytopathology 104:1125-1129.

Visser, M., Burger, J. T., and Maree, H. J. 2016. Targeted virus detection in next-generation sequencing data using an automated e-probe based approach. Virology 495:122-128. 